\title{
Determinantes antropométricos da pressão arterial elevada em escolares do ensino fundamental
}

\author{
Anthropometric determinants of high blood pressure in elementary schoolchildren \\ Determinantes antropométricos de la presión arterial alta en escuelas de educación fundamental
}

\author{
Laiza Santos Pimentel Haddada (D) Kiscila Araújo Fernandes ${ }^{a}$ (D) Guilherme Burini Lopes ${ }^{\mathrm{a}}$ (D), Francielle Bosi Rodrigues Veloso ${ }^{\mathrm{a}}$ (D),
} Sheila Cristina Caniçalia ${ }^{\circledR}$, Wanêssa Lacerda Poton ${ }^{a}$ (i)

aniversidade Vila Velha - Vila Velha (ES), Brasil.

\section{RESUMO}

Introdução: A hipertensão arterial infantil vem recebendo atenção especial dos pediatras, pois o aumento da pressão arterial na infância contribui para o início precoce da hipertensão arterial essencial na idade adulta e para a mortalidade por doenças cardiovasculares. As medidas antropométricas têm sido úteis para o diagnóstico de sobrepeso e obesidade na infância, e tais condições são consideradas de risco para hipertensão arterial na idade adulta. Quanto mais precoce a identificação desses fatores de risco, seja no ambiente escolar, seja nos serviços de saúde, mais ações preventivas poderão ser desenvolvidas para minimizar tal problemática. Objetivo: Identificar a incidência de pressão arterial elevada e sua associação com medidas antropométricas em escolares do ensino fundamental. Métodos: Estudo longitudinal com 1.116 escolares; destes, 133 participaram de três avaliações no período de 2017 a 2019. As informações demográficas, as medidas antropométricas (peso, altura, circunferência abdominal, índice de massa corporal) e as pressóricas (sistólica e diastólica ajustadas de acordo com os parâmetros do Centro de Controle e Prevenção de Doenças para sexo e idade) foram registradas em formulário. A associação das variáveis com a pressão arterial elevada foi analisada por meio da regressão de Poisson, com ajuste robusto da variância. Resultados: Dos estudantes, 51,6\% eram meninos com, em média, 7,9 anos, e $45,4 \%$ tinham pressão arterial elevada conforme os critérios do Centro de Controle e Prevenção de Doenças. Entre os que apresentaram circunferência abdominal elevada, 19,4\% evoluíram de pressão sistólica normal para elevada e $35,5 \%$ de pressão diastólica normal para elevada ao longo dos três anos de acompanhamento. Nos escolares com sobrepeso ou obesidade, a pressão sistólica normal evoluiu para elevada em 20,7 e $21,2 \%$, respectivamente, e a pressão diastólica normal evoluiu para elevada em 24,1 e $42,4 \%$, respectivamente. Os escolares com circunferência abdominal (risco relativo - RR 1,51; intervalo de confiança - IC95\% 1,20-1,91; RR 1,58; IC95\% 1,25-2,00), peso (RR 1,37; IC95\% 1,08-1,74; RR 1,34; IC95\% 1,05-1,71) e índice de massa corporal elevado (RR 1,51; IC95\% 1,21-1,87; RR 1,50; IC95\% 1,20-1,88) apresentaram maior risco para hipertensão sistólica e diastólica, respectivamente. Conclusão: A circunferência abdominal, o peso e o índice de massa corporal estiveram associados com o aumento da pressão arterial sistólica e diastólica nos escolares, e o risco foi maior entre os que tinham circunferência abdominal aumentada.

Palavras-chave: Criança. Sobrepeso. Obesidade pediátrica. Hipertensão. Doenças cardiovasculares.

\author{
Autor correspondente: \\ Laiza Santos Pimentel Haddad \\ E-mail: laizahaddad@ hotmail.com \\ Fonte de financiamento: \\ Programa Institucional de Bolsas de Inicia- \\ ção Científica, Universidade Vila Velha, Vila \\ Velha (ES), Brasil, no do processo 19/2017. \\ Parecer: \\ 1.751.120. \\ Procedência: \\ não encomendado. \\ Avaliação por pares: \\ externa. \\ Recebido em: 06/11/2020. \\ Aprovado em: 06/08/2021.
}

Como citar: Haddad LSP, Fernandes KA, Lopes GB, Veloso FBR, Caniçali SC, Poton WL. Determinantes 2021;16(43):2779. https://doi.org/10.5712/rbmfc16(43)2779; 


\begin{abstract}
Introduction: Pediatric hypertension has received special attention from pediatricians, as increased blood pressure in childhood contributes to the early onset of essential hypertension in adulthood and mortality from cardiovascular diseases. Anthropometric measurements have been useful in diagnosing childhood overweight and obesity, considered risk conditions for hypertension in adulthood. The earlier the identification of these risk factors, whether in the school environment or in health services, the higher the number of preventive actions that can be developed to reduce this problem. Objective: To identify the incidence of high blood pressure and its association with anthropometric measurements in elementary schoolchildren. Methods: This is a longitudinal study with 1,116 schoolchildren, 133 of whom participated in three assessments from 2017 to 2019. Demographic information, anthropometric measures (weight, height, waist circumference, body mass index), and blood pressure values (systolic and diastolic adjusted for sex and age according to parameters from the Centers for Disease Control and Prevention) were recorded in a form. The association of variables with high blood pressure was analyzed using Poisson regression, with robust variance adjustment. Results: A total of $51.6 \%$ of students were boys with a mean age of 7.9 years, and $45.4 \%$ had high blood pressure according to criteria from the Centers for Disease Control and Prevention. Among those who presented high waist circumference, 19.4\% progressed from normal to high systolic blood pressure, and $35.5 \%$ from normal to high diastolic blood pressure over the three years of follow-up. In overweight and obese schoolchildren, normal systolic blood pressure progressed to high in 20.7 and $21.2 \%$ of cases, respectively, and normal diastolic blood pressure progressed to high in 24.1 and $42.4 \%$, respectively. Schoolchildren with high waist circumference (relative risk - RR 1.51; confidence interval - 95\% $\mathrm{Cl} \mathrm{1.20-1.91;} \mathrm{RR} \mathrm{1.58;} \mathrm{95 \% Cl} \mathrm{1.25-2.00),}$ weight (RR 1.37; 95\%Cl 1.08-1.74; RR 1.34; 95\% Cl 1.05-1.71), and body mass index (RR 1.51; 95\%Cl 1.21-1.87; RR 1.50; 95\%Cl 1.20-1.88) presented a greater risk for systolic and diastolic hypertension, respectively. Conclusions: Waist circumference, weight, and body mass index were associated with increased systolic and diastolic blood pressure in schoolchildren, and the risk was greater among those with higher waist circumference.
\end{abstract}

Keywords: Child; Overweight; Pediatric obesity; Hypertension; Cardiovascular diseases.

\title{
RESUMEN
}

Introducción: La hipertensión arterial infantil ha recibido especial atención por parte de los pediatras, ya que el aumento de la presión arterial en la infancia contribuye a la aparición precoz de la hipertensión arterial esencial en la edad adulta y la mortalidad por enfermedades cardiovasculares. Las mediciones antropométricas han sido útiles para el diagnóstico de sobrepeso y obesidad en la infancia, y estas condiciones se consideran un riesgo de hipertensión arterial en la edad adulta. Cuanto antes se identifiquen estos factores de riesgo, ya sea en el ámbito escolar o en los servicios de salud, más acciones preventivas se pueden desarrollar para minimizar este problema. Objetivo: Identificar la incidencia de hipertensión arterial y su asociación con medidas antropométricas en estudiantes de primaria. Métodos: Estudio longitudinal con 1.116 estudiantes; de estos, 133 participaron en tres evaluaciones de 2017 a 2019. Información demográfica, medidas antropométricas (peso, talla, circunferencia de cintura, índice de masa corporal) y presión arterial (sistólica y diastólica ajustadas según los parámetros del Centro de Control y Prevención de Enfermedades, por sexo y edad) se registraron en un formulario. La asociación de variables con hipertensión arterial se analizó mediante regresión de Poisson con ajuste de varianza robusto. Resultados: El 51,6\% de los estudiantes era varón con un promedio de 7,9 años y el $45,4 \%$ presentaba hipertensión arterial, según los criterios del Centro para el Control y la Prevención de Enfermedades. Entre los que tenían una circunferencia de cintura alta, el 19,4\% evolucionó de presión sistólica normal a alta y el 35,5\% de presión diastólica normal a alta durante los tres años de seguimiento. En escolares con sobrepeso u obesidad, la presión sistólica normal evolucionó a alta en un $20,7 \%$ y un $21,2 \%$, respectivamente, y la presión diastólica normal evolucionó a alta en un $24,1 \%$ y un $42,4 \%$, respectivamente. Estudiantes con circunferencia de cintura (RR 1,51; IC95\% 1,20-1,91; RR 1,58; IC95\% 1,25-2,00), peso (RR 1,37; IC95\% 1,08-1,74; RR 1,34; IC95\% 1,05-1,71) y alto índice de masa corporal (RR 1,51; IC95\% 1,21-1,87; RR 1,50; IC95\% 1, 20-1,88) tenían un mayor riesgo de hipertensión sistólica y diastólica, respectivamente. Conclusiones: La circunferencia de la cintura, el peso y el índice de masa corporal se asociaron con un aumento de la presión arterial sistólica y diastólica en los escolares y el riesgo fue mayor entre los que tenían una mayor circunferencia de la cintura.

Palabras-clave: Niño. Sobrepeso. Obesidad pediátrica. Hipertensión. Enfermedades cardiovasculares.

\section{INTRODUÇÃO}

A identificação da hipertensão arterial infantil é de grande importância para a saúde pública, pois o aumento da pressão arterial $(\mathrm{PA})$ na infância contribui substancialmente para a mortalidade por doenças cardiovasculares na idade adulta. ${ }^{1}$ Estudos indicam que alterações ateroscleróticas podem iniciar-se na infância, ${ }^{1,2}$ assim como a hipertrofia do ventrículo esquerdo ${ }^{3} \mathrm{e}$ as disfunções miocárdicas. ${ }^{3}$ Soma-se a isso o fato de a presença da PA elevada na infância ser um fator preditor de hipertensão na vida adulta. ${ }^{2}$ Sendo assim, a falta do diagnóstico de hipertensão na infância promove um atraso ao início do tratamento da enfermidade, o que contribui para a sua persistência até a maioridade e aumenta a prevalência de repercussões da doença uma vez que a duração da hipertensão se relaciona ao risco de danos aos órgãos alvo. ${ }^{4}$ 
Nas últimas décadas, vem-se observando aumento na prevalência de hipertensão em crianças e adolescentes. ${ }^{4}$ Metanálise de estudos realizados na África encontrou prevalência de $12,7 \%$ de pré-hipertensão e 5,5\% de hipertensão em crianças e adolescentes de dois a 19 anos, ${ }^{5}$ resultado condizente com os de um estudo com estadunidenses de três a 18 anos de idade, dos quais 10,1\% tinham pré-hipertensão e 3,3\% hipertensão. ${ }^{6}$ Entretanto, outra pesquisa realizada nos Estados Unidos observou aumento de $38 \%$ na prevalência de pré-hipertensão e hipertensão em crianças e adolescentes com oito a 17 anos, mais frequente nos meninos $(19,2 \%)$ do que nas meninas $(12,6 \%) .{ }^{7}$ Na Europa há uma variação na prevalência de hipertensão, nessa população, de 2,2 a 22,0\%. ${ }^{8}$ No Brasil, uma pesquisa de base escolar observou que $9,6 \%$ dos adolescentes tinham hipertensão e esta condição foi mais frequente na região Sul do país (12,5\%). ${ }^{9}$ A variabilidade nas prevalências observadas nos estudos é explicada por diferenças no método de mensuração, incluindo o tipo de dispositivo utilizado e o número de medições, bem como algumas características da população, por exemplo, participantes com obesidade. ${ }^{5,8}$

Vários fatores de risco estão associados à hipertensão em crianças e adolescentes, sendo o excesso de peso o mais consistentemente documentado na gênese dessa doença. ${ }^{9}$ Diversos estudos vêm demonstrando que a prevalência de hipertensão em crianças e adolescentes está aumentando paralelamente ao aumento global na prevalência de sobrepeso e obesidade nessas fases da vida. . $^{9,10}$ No Brasil, de acordo com dados do Sistema de Vigilância Alimentar e Nutricional (SISVAN), 15,7\% das crianças entre cinco e dez anos de idade estão com sobrepeso, ao passo que 8,9\% estão obesas, e especificamente no Espírito Santo 16,2 e 8,7\% das crianças nessa faixa etária apresentam sobrepeso e obesidade, respectivamente. ${ }^{11}$ Em relação aos adolescentes brasileiros, 19,3\% estão com sobrepeso e 9,4\% estão obesos. ${ }^{11}$ Assim, um estudo transversal com 4.609 escolares do município de Maringá analisou a prevalência de obesidade e sua associação com o aumento da PA e identificou que a PA elevada ou limítrofe nos estudantes com excesso de peso foi quase duas vezes mais prevalente do que nos eutróficos. ${ }^{12}$

Como consequência, a hipertensão arterial relacionada ao excesso de peso na adolescência vem sendo cada vez mais investigada, pois nessa associação há um aumento potencial do risco de doença cardiovascular em relação a outro fator de risco isoladamente. ${ }^{13}$ Nesse sentido, a antropometria é amplamente utilizada na avaliação do estado nutricional infantil nos serviços de saúde dadas as suas vantagens, como: baixo custo, simplicidade de realização, facilidade de aplicação e padronização e amplitude dos aspectos analisados, além de ela não ser invasiva. ${ }^{14}$ Todavia, a literatura diverge quanto à magnitude das associações entre diferentes medidas antropométricas e o risco cardiometabólico em crianças. ${ }^{15}$ A associação entre gordura visceral, avaliada pela circunferência abdominal (CA), e a PA tem sido amplamente estudada em adultos, e observa-se que o aumento na CA eleva a PA. ${ }^{16}$ Entretanto, apesar de existirem evidências comprovando essa relação nos adultos, tal associação vem sendo recentemente investigada em crianças. ${ }^{17}$

Pesquisa realizada com 38.810 estudantes chineses, crianças e adolescentes, com baixo índice de massa corporal (IMC) e CA elevada indicou que eles apresentavam risco aumentado de hipertensão arterial, que seria subestimado ao se considerar apenas o IMC. ${ }^{18}$ Por outro lado, estudos mostraram uma forte correlação entre o aumento do percentil do IMC infantil com o risco de hipertensão. ${ }^{19}$

Considerando-se que o diagnóstico precoce de hipertensão na criança é importante para diminuir a morbimortalidade por doença cardiovascular na idade adulta e que as medidas antropométricas, acessíveis e de fácil realização, podem ser ferramentas muito úteis na identificação de sobrepeso e obesidade, o presente estudo objetivou identificar a incidência de hipertensão em escolares de uma escola do ensino 
fundamental ao longo de três anos, bem como analisar sua associação com as medidas antropométricas IMC e CA, que podem predizer risco para hipertensão arterial.

\section{MÉTODOS}

Este estudo longitudinal foi realizado uma escola pública municipal de ensino fundamental da cidade de Vila Velha, Espírito Santo, Brasil. Os acompanhamentos foram realizados durante três anos, de 2017 a 2019, por um grupo de estudantes do curso de Medicina selecionados e treinados, participantes do projeto de extensão "Saúde na Escola: um projeto de pesquisa-intervenção". Dessa forma, a escolha da escola ocorreu por conveniência, considerando-se que foram utilizados dados de um projeto de extensão já existente na escola em questão.

A amostra foi constituída de todos os escolares regularmente matriculados e cujos pais consentiram com a sua participação no projeto de extensão $(n=1.422)$.

A perda amostral foi de $21,5 \%$ e corresponde aos escolares que não obtiveram as medidas de PA, CA, peso ou altura registrados em algum dos três anos $(n=306)$. O poder da amostra foi de $100 \%$ para um erro tipo I $<5 \%$, incidência de pressão arterial elevada de $55 \%$ nos expostos e de $39 \%$ entre os não expostos e razão de incidências de 1,4.

Um formulário foi elaborado para registro anual das informações do estudante, no qual foram anotadas as medidas antropométricas, a PA e outras informações sobre a saúde do estudante. As informações sociodemográficas incluídas no questionário foram sexo, idade e cor da pele.

As variáveis utilizadas neste estudo foram: idade em anos e em categorias (6 a 7; 8 a $9 ; 10$ a 12), cor da pele (branca; não branca), peso em gramas e classificado de acordo com o sexo e a idade (baixo; adequado; elevado), CA em centímetros e classificada de acordo com o sexo e a idade (adequada; elevada), IMC em $\mathrm{kg} / \mathrm{m}^{2}$ e classificado de acordo com o sexo e a idade (magreza; eutrofia; sobrepeso; obesidade), pressão arterial sistólica (PAS) e pressão arterial diastólica (PAD) em mmHg e classificadas de acordo com o sexo, a idade e o escore da altura (normal; elevada).

O peso foi aferido em uma balança eletrônica portátil da marca Líder, com capacidade de até $200 \mathrm{~kg}$ e divisão de 50 gramas, calibrada e certificada pelo Instituto Nacional de Metrologia, Qualidade e Tecnologia (Inmetro). O estudante foi orientado a remover os sapatos, casaco e possíveis adornos e, em seguida, a ficar em pé com os dois pés no centro da balança, com as mãos ao lado e olhando para a frente. O peso foi registrado na fração decimal mais próxima e foi categorizado de acordo com o SISVAN, ${ }^{14}$ utilizando-se os gráficos de peso para idade e sexo. A classificação adotada foi:

1. Baixo peso: menor que percentil 3;

2. Peso adequado: percentil maior ou igual a 3 e menor que 97 ;

3. Elevado: percentil maior ou igual a $97 .^{19}$

Para a aferição da estatura, foi empregado estadiômetro de parede, marca MD, com faixa de medição de 0 a $200 \mathrm{~cm}$ e graduação de $1 \mathrm{~mm}$. O estudante permaneceu sem sapatos, roupas volumosas ou adornos de cabelo, sendo posicionado de forma ereta, de costas para o encosto vertical do estadiômetro. As nádegas, escápulas e cabeça foram posicionadas em contato com a fita do estadiômetro e os braços ficaram pendentes nas laterais do tronco, com as palmas voltadas para os membros inferiores as coxas. A cabeça do estudante foi mantida na posição de plano horizontal de Frankfurt, enquanto o examinador abaixava a barra horizontal firmemente na coroa da cabeça com pressão suficiente para comprimir o 
cabelo. ${ }^{14} \mathrm{~A}$ altura foi classificada, individualmente, de acordo com o percentil, para posterior utilização na classificação da PA conforme orientações do SISVAN. ${ }^{14}$

A CA foi mensurada por uma fita métrica inelástica graduada em milímetros, colocada no ponto médio entre o rebordo costal inferior e a crista ilíaca. ${ }^{20}$ Foram considerados com CA elevada os escolares cujos valores, de acordo com o sexo e a idade, estavam iguais ou maiores que o percentil 90 e com CA adequada os que se encontravam abaixo desse percentil, segundo os pontos de corte propostos por Freedman et al. ${ }^{20}$

O IMC foi calculado utilizando-se o peso corporal $(\mathrm{kg})$ no numerador e a altura $\left(\mathrm{m}^{2}\right)$ no denominador e classificado de acordo com tabelas e gráficos padronizados para sexo, idade, altura e peso, como padronizado pelo SISVAN. ${ }^{14}$ Os escolares classificados com magreza estavam com o IMC abaixo do percentil 3; os eutróficos apresentavam IMC maior ou igual ao percentil 3 e menor que 85; os com sobrepeso tinham o IMC igual ou superior ao percentil 85 e abaixo do 97; e os obesos tiveram o IMC igual ou superior ao percentil $97 .^{14}$

A PA foi obtida por meio da técnica auscultatória com esfigmomanômetro aneroide, devidamente calibrado, com manguito adequado à dimensão da circunferência do braço do estudante, e um estetoscópio colocado sobre o pulso da artéria braquial, proximal e medial à fossa cubital e abaixo da borda inferior do manguito. ${ }^{21} \mathrm{O}$ estudante era convocado para a aferição da PA antes do recreio e em horários distantes da aula de educação física, sendo instruído a permanecer por 5 min em repouso e sentado em ambiente calmo, com as pernas descruzadas e pés apoiados no chão, dorso recostado na cadeira, braço apoiado na mesma altura do coração, palma da mão voltada para cima e cotovelo ligeiramente fletido, em conformidade com as orientações do quarto relatório sobre diagnóstico, avaliação e tratamento da hipertensão arterial em crianças e adolescentes. ${ }^{21}$ A PAS foi determinada no início dos sons de Korotkoff (fase I) e a PAD no desaparecimento dos sons de Korotkoff (fase V). A PA foi classificada de acordo com o percentil de altura, idade e sexo em virtude de sua relação com o tamanho corporal. ${ }^{14}$ Assim, o estudante foi considerado normotenso quando a PA foi menor que o percentil 90 e com PA elevada quando ela foi maior ou igual ao percentil 90. A medição foi repetida em pelo menos duas ocasiões adicionais para confirmar o diagnóstico. ${ }^{21}$

As informações dos questionários foram digitadas no software Microsoft Excel ${ }^{\circledR}$ e as análises estatísticas conduzidas no programa estatístico Stata, versão 13.0 (StataCorp LP, College Station, Estados Unidos). Foram selecionados os estudantes que participaram de pelo menos um dos acompanhamentos e que possuíam todos os dados registrados $(n=1.116)$. As variáveis foram estratificadas por sexo. Entretanto, a única diferença encontrada entre os sexos foi em relação à idade, por isso a análise de associação não foi realizada separadamente. Para variáveis categóricas foi aplicado o teste qui-quadrado de heterogeneidade e o resultado foi apresentado como frequência simples e relativa. As variáveis numéricas foram apresentadas como média e desvio padrão e aplicouse o teste $t$ de Student. A regressão de Poisson com ajuste robusto da variância foi empregada para verificar a razão de risco da PAS e da PAD com as variáveis exploratórias, considerando-se o intervalo de confiança de $95 \%$.

O termo de consentimento livre e esclarecido foi aprovado pelo Comitê de Ética em Pesquisa da Universidade Vila Velha (parecer $n^{\circ} 1.751 .120$ ), entregue aos estudantes e assinado pelos pais ou responsáveis. Um termo de autorização para a realização do projeto foi obtido com a direção da escola. A pesquisa foi registrada na Plataforma Brasil ( $n^{\circ}$ de registro: 79143417.0.0000.5064) e iniciou-se após ciência e anuência da escola e dos pais ou responsáveis. 


\section{RESULTADOS}

Dos 1.116 escolares, a maioria era de meninos (51,6\%) com, em média, 7,9 anos de idade e cor da pele não branca $(66,4 \%)$. A cada cinco escolares acompanhados, um tinha peso e/ou CA elevada e em torno de dois apresentavam sobrepeso ou obesidade pelo IMC. Um quarto dos escolares estava com a PAS ou a PAD elevada. A única diferença observada entre meninos e meninas foi em relação à idade, já que as meninas eram mais jovens $(p=0,008)$ (Tabela 1$)$.

A Tabela 2 apresenta as mudanças na classificação da PAS em 133 escolares acompanhados em dois momentos (2017 e 2019). Quase metade dos escolares com sobrepeso ou obesidade tiveram mudanças na PAS de normal para elevada e pouco mais de 15\% passaram de PAS elevada para normal. Dos escolares com obesidade e dos eutróficos, respectivamente 21,2 e 4,2\% mantiveram-na elevada nos dois acompanhamentos $(p=0,005)$.

Em relação à PAD (Tabela 3 ), mais da metade dos escolares com peso normal e com sobrepeso mantiveram-na normal e mais de $40 \%$ dos obesos tiveram mudanças nessa medida de normal para elevada. Somente $6,1 \%$ dos obesos modificaram-na de elevada para normal e quase um quinto dos escolares com sobrepeso e obesidade mantiveram-na elevada nos dois acompanhamentos $(p=0,010)$.

A Tabela 4 apresenta o efeito das variáveis independentes sobre a PAS e a PAD. Os valores pressóricos sistólico e diastólico foram menores nos escolares de oito a nove anos de idade (risco relativo - RR 0,62; intervalo de confiança - IC95\% 0,48-0,80; RR 0,62; IC95\% 0,48-0,81) comparados aos de seis e sete anos. A prevalência de PAS elevada foi maior nos escolares com CA (RR 1,51; IC95\% 1,20-1,91), peso (RR 1,37; IC95\% 1,08-1,74) e IMC (RR 1,51; IC95\% 1,21-1,87) elevados, comparados aos que apresentavam essas medidas normais. A prevalência de PAD elevada foi maior nos escolares com CA (RR 1,58; IC95\% 1,25-2,00), peso (RR 1,34; IC95\% 1,05-1,71) e IMC elevados (RR 1,50; IC95\% 1,20-1,88). Observou-se também uma interação entre as PAS e PAD de forma concomitante, demonstrando que o aumento de uma variável influencia no aumento da outra $(p<0,001)$.

\section{DISCUSSÃO}

Este estudo evidenciou que o peso, a CA e o IMC elevados estiveram associados com o aumento da PAS e da PAD, porém a CA elevada apresentou associação mais forte que o IMC. Por outro lado, ao se analisar a evolução da PA ao longo dos anos, apesar de os resultados irem na mesma direção ou seja, identificando forte relação de CA com o aumento da PA —, quase metade dos escolares com sobrepeso ou obesidade evoluíram de PAS normal para PAS elevada e mais da metade de PAD normal para elevada.

Diversos estudos investigaram a relação da PA com os índices antropométricos em estudantes de diferentes países. ${ }^{14,22}$ Pesquisa realizada em Taiwan, com 2.334 crianças de seis a sete anos, encontrou prevalência de PA elevada em meninos e meninas que estavam no quarto quartil da CA (38,9 e 26,8\%, respectivamente). ${ }^{23}$ No Brasil, 3.417 crianças com IMC normal foram avaliadas e o aumento da CA esteve associado à PA elevada, ou seja, mesmo quando o IMC era normal. ${ }^{24}$ Isso vai ao encontro do resultado obtido no presente estudo, no qual a CA apresentou associação positiva com a hipertensão na infância.

Em contrapartida, outras pesquisas enfatizaram a relação do IMC com a PA elevada. ${ }^{10,25} \mathrm{O}$ estudo longitudinal de Fels sugere que níveis aumentados de PA na idade adulta possam ter sua gênese no 
Tabela 1. Distribuição dos escolares estratificados por sexo, segundo idade, cor da pele, peso, circunferência abdominal, índice de massa corporal, pressão arterial sistólica e diastólica, Vila Velha (ES), 2017 a 2019 (n=1.116)

\begin{tabular}{|c|c|c|c|c|c|c|c|}
\hline & \multicolumn{2}{|c|}{$\begin{array}{c}\text { Masculino } \\
\mathrm{n}=576\end{array}$} & \multicolumn{2}{|c|}{$\begin{array}{c}\text { Feminino } \\
n=540\end{array}$} & \multicolumn{2}{|c|}{$\begin{array}{c}\text { Total } \\
n=1.116\end{array}$} & Valor $p$ \\
\hline Idade (anos) & \multicolumn{2}{|c|}{$8,1 \pm 1,5$} & \multicolumn{2}{|c|}{$7,8 \pm 1,4$} & \multicolumn{2}{|c|}{$7,9 \pm 1,5$} & $0,008^{*}$ \\
\hline $6-7$ & 234 & $\begin{array}{c}40,6 \% \\
(36,7-44,7)\end{array}$ & 251 & $\begin{array}{c}46,5 \% \\
(42,3-50,7)\end{array}$ & 485 & $\begin{array}{c}43,4 \% \\
(40,6-46,4)\end{array}$ & \\
\hline $8-9$ & 214 & $\begin{array}{c}37,2 \% \\
(33,3-41,2)\end{array}$ & 189 & $\begin{array}{c}35,0 \% \\
(31,1-39,1)\end{array}$ & 403 & $\begin{array}{c}36,1 \% \\
(33,3-39,0)\end{array}$ & $0,110^{+}$ \\
\hline $10-12$ & 128 & $\begin{array}{c}22,2 \% \\
(19,0-25,8)\end{array}$ & 100 & $\begin{array}{c}18,5 \% \\
(15,4-22,0)\end{array}$ & 228 & $\begin{array}{c}20,4 \% \\
(18,2-22,9)\end{array}$ & \\
\hline \multicolumn{8}{|l|}{ Cor da pele } \\
\hline Branca & 139 & $\begin{array}{c}34,0 \% \\
(29,5-38,7)\end{array}$ & 129 & $\begin{array}{c}33,2 \% \\
(28,6-38,0)\end{array}$ & 268 & $\begin{array}{c}33,6 \% \\
(30,4-36,9)\end{array}$ & $0,822^{\dagger}$ \\
\hline Não branca & 270 & $\begin{array}{c}66,0 \% \\
(61,3-70,5)\end{array}$ & 260 & $\begin{array}{c}66,8 \% \\
(62,0-71,4)\end{array}$ & 530 & $\begin{array}{c}66,4 \% \\
(63,0-69,6)\end{array}$ & \\
\hline Peso (kg) & \multicolumn{2}{|c|}{$31,4 \pm 9,5$} & \multicolumn{2}{|c|}{$31,4 \pm 10,4$} & \multicolumn{2}{|c|}{$31,4 \pm 9,9$} & $0,931^{*}$ \\
\hline Baixo & 10 & $\begin{array}{c}1,7 \% \\
(0,9-3,2)\end{array}$ & 6 & $\begin{array}{c}1,1 \% \\
(0,5-2,4)\end{array}$ & 16 & $\begin{array}{c}1,4 \% \\
(0,9-2,3)\end{array}$ & \\
\hline Adequado & 453 & $\begin{array}{c}78,7 \% \\
(75,1-81,8)\end{array}$ & 422 & $\begin{array}{c}78,2 \% \\
(74,4-81,4)\end{array}$ & 875 & $\begin{array}{c}78,4 \% \\
(75,9-80,7)\end{array}$ & $0,630^{\dagger}$ \\
\hline Elevado & 113 & $\begin{array}{c}19,6 \% \\
(16,6-23,1)\end{array}$ & 112 & $\begin{array}{c}20,7 \% \\
(17,5-24,4)\end{array}$ & 225 & $\begin{array}{c}20,2 \% \\
(17,9-22,6)\end{array}$ & \\
\hline $\mathrm{CA}(\mathrm{cm})$ & \multicolumn{2}{|c|}{$63,2 \pm 24,7$} & \multicolumn{2}{|c|}{$61,5 \pm 10,4$} & \multicolumn{2}{|c|}{$62,4 \pm 19,2$} & $0,145^{*}$ \\
\hline Adequada & 460 & $\begin{array}{c}79,9 \% \\
(76,4-82,9)\end{array}$ & 435 & $\begin{array}{c}80,6 \% \\
(77,0-83,7)\end{array}$ & 895 & $\begin{array}{c}80,2 \% \\
(77,7-82,4)\end{array}$ & ח 820? \\
\hline Elevada & 116 & $\begin{array}{c}20,1 \% \\
(17,0-23,6)\end{array}$ & 105 & $\begin{array}{c}19,4 \% \\
(16,3-23,0)\end{array}$ & 221 & $\begin{array}{c}19,8 \% \\
(17,6-22,2)\end{array}$ & $0,0<2$ \\
\hline IMC $\left(\mathrm{kg} / \mathrm{m}^{2}\right)$ & \multicolumn{2}{|c|}{$17,6 \pm 3,4$} & \multicolumn{2}{|c|}{$17,7 \pm 3,7$} & \multicolumn{2}{|c|}{$17,6 \pm 3,5$} & $0,618^{*}$ \\
\hline Magreza & 28 & $\begin{array}{c}4,9 \% \\
(3,4-6,9)\end{array}$ & 15 & $\begin{array}{c}2,8 \% \\
(1,7-4,6)\end{array}$ & 43 & $\begin{array}{c}3,9 \% \\
(2,9-5,2)\end{array}$ & \\
\hline Eutrofia & 316 & $\begin{array}{c}54,9 \% \\
(50,8-58,9)\end{array}$ & 325 & $\begin{array}{c}60,2 \% \\
(56,0-64,2)\end{array}$ & 641 & $\begin{array}{c}57,4 \% \\
(54,5-60,3)\end{array}$ & \\
\hline Sobrepeso & 103 & $\begin{array}{c}17,8 \% \\
(14,9-21,2)\end{array}$ & 101 & $\begin{array}{c}18,7 \% \\
(15,6-22,2)\end{array}$ & 204 & $\begin{array}{c}18,3 \% \\
(16,1-20,7)\end{array}$ & $0,076^{\dagger}$ \\
\hline Obesidade & 129 & $\begin{array}{c}22,4 \% \\
(19,2-26,0)\end{array}$ & 99 & $\begin{array}{c}18,3 \% \\
(15,3-21,8)\end{array}$ & 228 & $\begin{array}{c}20,4 \% \\
(18,2-22,9)\end{array}$ & \\
\hline PAS (mmHg) & \multicolumn{2}{|c|}{$99,3 \pm 13,8$} & \multicolumn{2}{|c|}{$101,7 \pm 58,9$} & \multicolumn{2}{|c|}{$100,5 \pm 42,2$} & $0,360^{*}$ \\
\hline Normal & 446 & $\begin{array}{c}77,4 \% \\
(73,8-80,7)\end{array}$ & 413 & $\begin{array}{c}76,5 \% \\
(72,7-79,9)\end{array}$ & 859 & $\begin{array}{c}77,0 \% \\
(74,4-79,3)\end{array}$ & \\
\hline Elevada & 130 & $\begin{array}{c}22,6 \% \\
(19,3-26,2)\end{array}$ & 127 & $\begin{array}{c}23,5 \% \\
(20,1-27,3)\end{array}$ & 257 & $\begin{array}{c}23,0 \% \\
(20,6-25,6)\end{array}$ & $0,722^{\dagger}$ \\
\hline PAD (mmHg) & \multicolumn{2}{|c|}{$65,0 \pm 10,9$} & \multicolumn{2}{|c|}{$65,4 \pm 27,8$} & \multicolumn{2}{|c|}{$65,2 \pm 20,8$} & $0,753^{*}$ \\
\hline Normal & 457 & $\begin{array}{c}79,4 \% \\
(75,8-82,5)\end{array}$ & 410 & $\begin{array}{c}75,9 \% \\
(72,1-79,3)\end{array}$ & 867 & $\begin{array}{c}77,7 \% \\
(75,1-80,0)\end{array}$ & \\
\hline Elevada & 119 & $\begin{array}{c}20,6 \% \\
(17,5-24,2)\end{array}$ & 130 & $\begin{array}{c}24,1 \% \\
(20,6-27,9)\end{array}$ & 249 & $\begin{array}{c}22,3 \% \\
(20,0-24,8)\end{array}$ & $0,173^{\dagger}$ \\
\hline
\end{tabular}

CA: circunferência abdominal; IMC: índice de massa corporal; PAS: pressão arterial sistólica; PAD: pressão arterial diastólica. ${ }^{*}$ Teste $t$; †Teste qui-quadrado; ${ }^{\ddagger}$ Variável não incluída no acompanhamento de 2017 (n=798). 
Tabela 2. Mudança da pressão arterial sistólica dos escolares, de acordo com o sexo, cor da pele, peso, circunferência abdominal, índice de massa corporal, Vila Velha (ES), 2017 a 2019 ( $n=133)$.

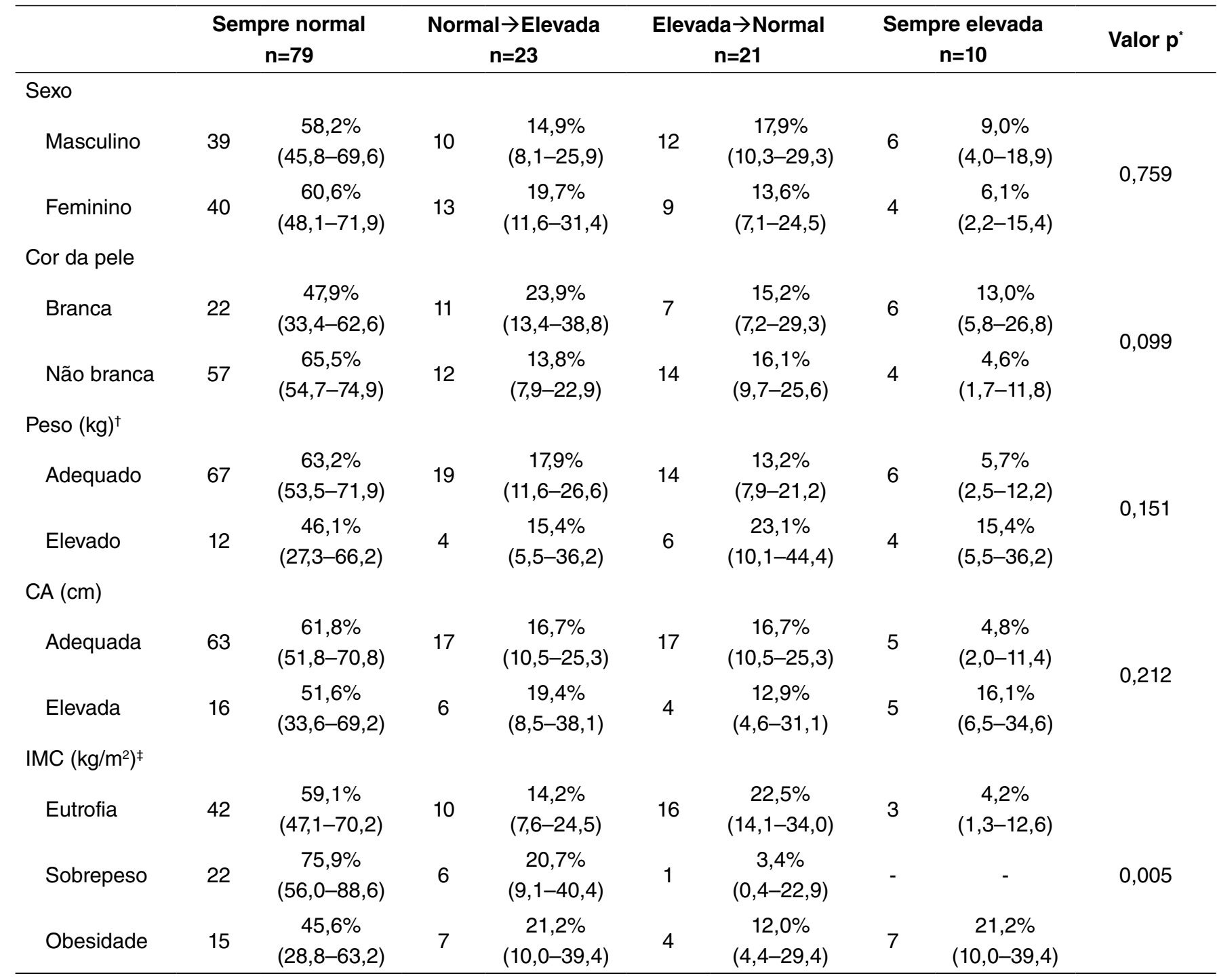

CA: circunferência abdominal; IMC: índice de massa corporal. A verificação da mudança na categoria da pressão arterial deu-se

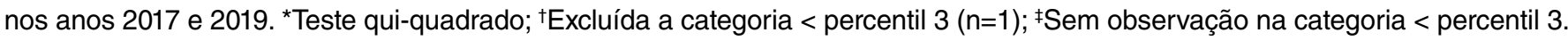

desenvolvimento da adiposidade infantil, expressa pelo IMC, visto que os estudantes com o maior IMC na infância foram os que desenvolveram altos níveis de PA na idade adulta, independentemente do sexo do participante e do próprio IMC quando adulto. ${ }^{26}$ Nesse sentido, o estudo destaca a adiposidade infantil como um dos fatores relacionados ao desenvolvimento da hipertensão arterial sistêmica (HAS) na vida adulta. ${ }^{26}$ Essa descoberta corrobora os resultados do presente estudo, já que mais da metade dos estudantes com IMC alterado (com sobrepeso ou obesos) e com PAS normal evoluíram para o aumento nos níveis pressóricos ou permaneceram com a PA elevada ao longo dos anos. Entretanto, é importante ressaltar que estudos recentes indicam que a puberdade precoce foi positivamente associada ao aumento do $\mathrm{IMC}^{27}$ e que o estágio puberal tem um efeito de interação na associação entre o risco cardiometabólico e o IMC..$^{28}$ 
Tabela 3. Mudança da pressão arterial diastólica dos escolares de acordo com o sexo, cor da pele, peso, circunferência abdominal, índice de massa corporal, Vila Velha (ES), 2017 a 2019 ( $n=133)$.

\begin{tabular}{|c|c|c|c|c|c|c|c|c|c|}
\hline Sexo & \multicolumn{2}{|c|}{$\begin{array}{c}\text { Sempre normal } \\
\mathrm{n}=72\end{array}$} & \multicolumn{2}{|c|}{$\begin{array}{c}\text { Normal } \rightarrow \text { Elevada } \\
n=38\end{array}$} & \multicolumn{2}{|c|}{$\begin{array}{c}\text { Elevada } \rightarrow \text { Normal } \\
n=15\end{array}$} & \multicolumn{2}{|c|}{$\begin{array}{c}\text { Sempre elevada } \\
n=8\end{array}$} & Valor $p^{*}$ \\
\hline Masculino & 37 & $\begin{array}{c}55,2 \% \\
(42,9-66,9)\end{array}$ & 18 & $\begin{array}{c}26,9 \% \\
(17,4-39,0)\end{array}$ & 10 & $\begin{array}{c}14,9 \% \\
(8,1-25,9)\end{array}$ & 2 & $\begin{array}{c}3,0 \% \\
(0,7-11,5)\end{array}$ & 0200 \\
\hline Feminino & 35 & $\begin{array}{c}53,0 \% \\
(40,7-65,0)\end{array}$ & 20 & $\begin{array}{c}30,3 \% \\
(20,2-42,7)\end{array}$ & 5 & $\begin{array}{c}7,6 \% \\
(3,1-17,3)\end{array}$ & 6 & $\begin{array}{c}9,1 \% \\
(4,0-19,1)\end{array}$ & 0,302 \\
\hline \multicolumn{10}{|l|}{ Cor da pele } \\
\hline Branca & 22 & $\begin{array}{c}47,8 \% \\
(33,4-62,6)\end{array}$ & 18 & $\begin{array}{c}39,1 \% \\
(25,8-54,3)\end{array}$ & 4 & $\begin{array}{c}8,7 \% \\
(3,2-21,6)\end{array}$ & 2 & $\begin{array}{c}4,4 \% \\
(1,0-16,5)\end{array}$ & \multirow{2}{*}{0,292} \\
\hline Não branca & 50 & $\begin{array}{c}57,5 \% \\
(46,7-67,6)\end{array}$ & 20 & $\begin{array}{c}23,0 \% \\
(15,2-33,2)\end{array}$ & 11 & $\begin{array}{c}12,6 \% \\
(7,1-21,6)\end{array}$ & 6 & $\begin{array}{c}6,9 \% \\
(3,1-14,7)\end{array}$ & \\
\hline \multicolumn{10}{|l|}{ Peso $(\mathrm{kg})^{\dagger}$} \\
\hline Adequado & 60 & $\begin{array}{c}56,6 \% \\
(46,9-65,8)\end{array}$ & 30 & $\begin{array}{c}28,3 \% \\
(20,4-37,7)\end{array}$ & 11 & $\begin{array}{c}10,4 \% \\
(5,8-17,9)\end{array}$ & 5 & $\begin{array}{c}4,7 \% \\
(1,9-11,0)\end{array}$ & \multirow{2}{*}{0,331} \\
\hline Elevado & 11 & $\begin{array}{c}42,3 \% \\
(24,2-62,8)\end{array}$ & 8 & $\begin{array}{c}30,8 \% \\
(15,4-52,0)\end{array}$ & 4 & $\begin{array}{c}15,4 \% \\
(5,5-36,3)\end{array}$ & 3 & $\begin{array}{c}11,5 \% \\
(3,5-32,1)\end{array}$ & \\
\hline \multicolumn{10}{|l|}{$\mathrm{CA}(\mathrm{cm})$} \\
\hline Adequada & 58 & $\begin{array}{c}56,9 \% \\
(46,9-66,3)\end{array}$ & 27 & $\begin{array}{c}26,5 \% \\
(18,7-36,0)\end{array}$ & 12 & $\begin{array}{c}11,7 \% \\
(6,7-19,7)\end{array}$ & 5 & $\begin{array}{c}4,9 \% \\
(2,0-11,4)\end{array}$ & \multirow{2}{*}{0,505} \\
\hline Elevada & 14 & $\begin{array}{c}45,1 \% \\
(28,0-63,5)\end{array}$ & 11 & $\begin{array}{c}35,5 \% \\
(20,1-54,5)\end{array}$ & 3 & $\begin{array}{c}9,7 \% \\
(2,9-27,4)\end{array}$ & 3 & $\begin{array}{c}9,7 \% \\
(2,9-27,4)\end{array}$ & \\
\hline \multicolumn{10}{|l|}{ IMC $\left(\mathrm{kg} / \mathrm{m}^{2}\right)^{\ddagger}$} \\
\hline Eutrofia & 39 & $\begin{array}{c}54,9 \% \\
(43,0-66,3)\end{array}$ & 17 & $\begin{array}{c}24,0 \% \\
(15,2-35,5)\end{array}$ & 13 & $\begin{array}{c}18,3 \% \\
(10,8-29,3)\end{array}$ & 2 & $\begin{array}{c}2,8 \% \\
(0,7-10,9)\end{array}$ & \multirow{3}{*}{0,010} \\
\hline Sobrepeso & 20 & $\begin{array}{c}69,0 \% \\
(49,0-83,7)\end{array}$ & 7 & $\begin{array}{c}24,1 \% \\
(11,4-44,0)\end{array}$ & - & - & 2 & $\begin{array}{c}6,9 \% \\
(1,6-25,4)\end{array}$ & \\
\hline Obesidade & 13 & $\begin{array}{c}39,4 \% \\
(23,7-57,6)\end{array}$ & 14 & $\begin{array}{c}42,4 \% \\
(26,2-60,4)\end{array}$ & 2 & $\begin{array}{c}6,1 \% \\
(1,4-22,6)\end{array}$ & 4 & $\begin{array}{c}12,1 \% \\
(4,4-29,4)\end{array}$ & \\
\hline
\end{tabular}

CA: circunferência abdominal; IMC: índice de massa corporal. A verificação da mudança na categoria da pressão arterial deu-se nos anos 2017 e 2019. *Teste qui-quadrado; ${ }^{\dagger}$ Excluída a categoria < percentil $3(n=1)$; ‘Sem observação na categoria < percentil 3.

Entre os mecanismos apontados na literatura para explicar a relação entre a PA elevada e a CA, considera-se que esta última é uma medida altamente sensível e específica da gordura da parte superior do corpo, a qual torna os indivíduos mais suscetíveis às doenças cardiovasculares e à síndrome metabólica, visto que a obesidade central pode refletir com precisão a extensão da gordura visceral. ${ }^{29}$ Embora o aumento da CA seja bem estabelecido como fator de risco para síndrome metabólica e doenças cardiovasculares na idade adulta, estudos em crianças ainda são limitados. ${ }^{29}$ Entretanto, uma pesquisa realizada com 12.043 estudantes de Minneapolis identificou que indivíduos com síndrome metabólica na infância apresentavam mais fatores de risco cardiovascular na idade adulta. ${ }^{30}$ Contudo, são necessários novos estudos para melhor compreensão sobre a relação entre a CA e o aumento da PA infantil. 
Tabela 4. Análise da associação do efeito das variáveis independentes com a pressão arterial sistólica e diastólica, Vila Velha (ES), 2017 a 2019 ( $n=1.116)$.

\begin{tabular}{|c|c|c|c|c|c|}
\hline & & & & & \\
\hline & & RR (IC95\%) & Valor $p$ & RR (IC95\%) & Valor $p$ \\
\hline Soyo & Masculino & Referência & 0707 & Referência & 0170 \\
\hline$s e x 0$ & Feminino & $1,04(0,84-1,29)$ & 0,701 & $1,16(0,03-1,45)$ & $0,1 / 2$ \\
\hline & $6-7$ & Referência & & Referência & \\
\hline Idade (anos) & $8-9$ & $0,62(0,48-0,80)$ & $<0,001$ & $0,62(0,48-0,81)$ & $<0,001$ \\
\hline & $10-12$ & $0,76(0,57-1,01)$ & & $0,69(0,51-0,94)$ & \\
\hline Cor da mele* & Branca & Referência & 0137 & Referência & 0678 \\
\hline tor ha peic & Não branca & $0,83(0,65-1,06)$ & 0,131 & $0,95(0,75-1,21)$ & $0,6 / 8$ \\
\hline $\operatorname{Pes}(k g)$ & Adequado & Referência & ( & Referência & מכח ח \\
\hline 1000 (ng) & Elevado & $1,37(1,08-1,74)$ & 0,000 & $1,34(1,05-1,71)$ & $v, v<v$ \\
\hline $\mathrm{CA}(\mathrm{cm})$ & Normal & Referência & $10 \cap 01$ & Referência & O००01 \\
\hline$U A(\mathrm{Cm})$ & Elevada & $1,51(1,20-1,91)$ & $<0,001$ & $1,58(1,25-2,00)$ & $<0,001$ \\
\hline$M C\left(\mathrm{~kg} / \mathrm{m}^{2}\right)$ & Normal & Referência & $<0001$ & Referência & ค م०1 \\
\hline 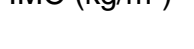 & Elevado & $1,51(1,21-1,87)$ & - & $1,50(1,20-1,88)$ & , \\
\hline$P A{ }^{t}$ & Normal & Referência & $<0001$ & Referência & $<0001$ \\
\hline 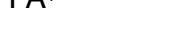 & Elevada & $9,68(7,62-12,29)$ & $<0,001$ & $10,53(8,16-13,58)$ & $\langle 0,001$ \\
\hline
\end{tabular}

PAS: pressão arterial sistólica; PAD: pressão arterial diastólica; RR: risco relativo; CA: circunferência abdominal; IMC: índice de massa corporal; PA: pressão arterial.

*Variável não incluída no acompanhamento de 2017 (n=798); †Comparação da PAS com a PAD e vice-versa.

Diferentemente, o Bogalusa Heart Study, estudo realizado com 11.478 crianças e adolescentes (de cinco a 17 anos), constatou que os níveis de PAS e PAD na amostra não aumentaram apesar do aumento na prevalência de obesidade, o que indica que o aumento no IMC em uma população não necessariamente estará relacionado à alteração nos níveis pressóricos. ${ }^{31}$ Adiciona-se a esse resultado um estudo de duas coortes de nascimentos britânicas, no qual a PA média não aumentou entre duas gerações nascidas com 12 anos de diferença, apesar dos níveis mais altos de IMC. Isso sugere que melhorias em outros fatores de risco ligados à PA na idade adulta, como tabagismo, consumo de sal e atividade física, ou mesmo características da amostra (como peso ao nascer e estatura média) podem, de certa forma, ter influenciado no resultado comparativo entre as coortes. ${ }^{10}$

A interação entre a PAS e a PAD também deve ser considerada. Observou-se, neste estudo, que $76 \%$ dos estudantes com PAS elevada também apresentavam PAD elevada. A Academia Americana de Pediatria indicou que a elevação da PAD está mais relacionada à hipertensão secundária, enquanto a hipertensão sistólica parece ser mais preditiva de HAS primária. ${ }^{32}$ Além disso, é sabido que quanto mais jovem a criança maior a chance de se tratar a hipertensão arterial secundária. ${ }^{32}$ As nefropatias parenquimatosas, renovasculares e obstrutivas são responsáveis por aproximadamente 60 a $90 \%$ desses casos, podendo acometer todas as faixas etárias (infantes, crianças e adolescentes), mas são mais incidentes em crianças mais jovens e com maiores elevações da PA. ${ }^{33}$ Os distúrbios endócrinos, como o excesso de mineralocorticoide, corticoide ou catecolaminas, as doenças da tireoide e a hipercalcemia 
associada ao hiperparatireoidismo correspondem a aproximadamente $5 \%$ dos casos. ${ }^{33} \mathrm{O}$ resultado encontrado, portanto, indica a necessidade de uma investigação detalhada, com história clínica e exame físico completo para a diferenciação dos casos de hipertensão primária e secundária. Isso porque a medida da PA ainda não é rotina na maioria dos exames feitos nas crianças brasileiras e as causas secundárias podem passar despercebidas, conforme indica a Sociedade Brasileira de Pediatria. ${ }^{34}$

É importante ressaltar algumas limitações deste estudo. Entre elas, destaca-se que foram avaliados estudantes de uma única instituição, logo a amostra pode não representar integralmente os escolares do município ou estado tendo em vista os determinantes sociais, principalmente as características sociodemográficas, as quais não foram investigadas neste estudo. Ademais, não houve dupla verificação da aferição da PA e nem todos tiveram os dados coletados mais de uma vez em momentos distintos. No entanto, mesmo com as perdas, foi encontrada uma forte relação entre a CA e o IMC com a hipertensão. Por fim, não foi analisado o estágio do desenvolvimento puberal das crianças, sendo possível que tenha ocorrido uma influência positiva do desenvolvimento puberal nos índices antropométricos no resultado da hipertensão arterial infantil, especialmente nas meninas.

Entre os pontos fortes, ressalta-se que o presente estudo analisou uma medida antropométrica ainda pouco investigada na infância e adolescência, a CA. Para a redução do viés de informação foram utilizados instrumentos de avaliação certificados e calibrados, conforme preconizado, o que garante a veracidade e a confiabilidade dos dados. Todos os pesquisadores de campo foram treinados para assegurar a validade das medidas antropométricas e pressóricas.

\section{CONCLUSÃO}

Os resultados sugerem que tanto os escolares com sobrepeso/obesidade como os que apresentam CA aumentada possuem níveis pressóricos mais elevados, o que se torna um risco para que esses indivíduos sejam adultos hipertensos e, consequentemente, contribuam para o desenvolvimento de doenças cardiovasculares. Assim, espera-se que tais resultados estimulem a realização de estudos de base populacional que analisem mais minuciosamente a relação da obesidade na infância e adolescência com a PA e que reforcem a necessidade de medidas de acompanhamento e controle da PA em crianças e adolescentes. Além disso, a inclusão de temas paralelos no currículo escolar da educação infantil, tais como alimentação saudável, atividade física e redução do tempo de tela, é outra ação importante no enfrentamento de tal problemática.

\section{CONFLITO DE INTERESSES}

Nada a declarar.

\section{CONTRIBUIÇÃO DOS AUTORES}

LSPH: administração do projeto, curadoria de dados, escrita - primeira redação, metodologia, escrita - revisão e edição. KAF: curadoria de dados, escrita - primeira redação, metodologia. GBL: curadoria de dados, escrita - primeira redação. FBRV: investigação, conceituação. SCC: investigação, conceituação. WLP: administração do projeto, análise formal, conceituação, escrita - revisão e edição, software, supervisão, validação e visualização. 


\section{REFERÊNCIAS}

1. Olson M, Chambers M, Shaibi G. Pediatric markers of adult cardiovascular disease. Curr Pediatr Rev 2017;13(4):255-9. https://doi.org/10.2174/1573396314666180117092010

2. Falkner B. The childhood role in development of primary hypertension. Am J Hypertens 2018;31(7):762-9. https://doi. org/10.1093/ajh/hpy058

3. Gupta-Malhotra M, Hamzeh RK, Poffenbarger T, McNiece-Redwine K, Hashmi SS. Myocardial performance index in childhood onset essential hypertension and white coat hypertension. Am J Hypertens 2016;29(3):379-87. https://doi.org/10.1093/ajh/ hpv123

4. Wühl E. Hypertension in childhood obesity. Acta Paediatr 2019;108(1):37-43. https://doi.org/10.1111/apa.14551

5. Noubiap JJ, Essouma M, Bigna JJ, Jingi AM, Aminde LN, Nansseu JR. Prevalence of elevated blood pressure in children and adolescents in Africa: a systematic review and meta-analysis. Lancet Public Health 2017;2(8):e375-e386. https://doi. org/10.1016/S2468-2667(17)30123-8

6. Kaelber DC, Liu W, Ross M, Localio AR, Leon JB, Pace WD, et al. Diagnosis and medication treatment of pediatric hypertension: a retrospective cohort study. Pediatrics 2016;138(6):e20162195. https://doi.org/10.1542/peds.2016-2195

7. Rosner B, Cook NR, Daniels S, Falkner B. Childhood blood pressure trends and risk factors for high blood pressure: the NHANES experience 1988-2008. Hypertension 2013;62(2):247-54. https://doi.org/10.1161/HYPERTENSIONAHA.111.00831

8. Lurbe E, Agabiti-Rosei E, Cruickshank JK, Dominiczak A, Erdine S, Hirth A, et al. 2016 European Society of Hypertension guidelines for the management of high blood pressure in children and adolescents. J Hypertens 2016;34(10):1887-920. https://doi.org/10.1097/HJH.0000000000001039

9. Bloch KV, Klein CH, Szklo M, Kuschnir MCC, Abreu GA, Barufaldi LA, et al. ERICA: prevalences of hypertension and obesity in Brazilian adolescents. Rev Saude Publica 2016;50(Suppl 1):9s. https://doi.org/10.1590/S01518-8787.2016050006685

10. Li L, Hardy R, Kuh D, Power C. Life-course body mass index trajectories and blood pressure in mid life in two British birth cohorts: stronger associations in the later-born generation. Int J Epidemiol 2015;44(3):1018-26. https://doi.org/10.1093/ije/ dyv106

11. Brasil. Ministério da Saúde. Sistema de Vigilância Alimentar e Nutricional. Relatórios de Acesso Público. Selecione o tipo de relatório [Internet] 2021. [acessado em 16 nov. 2021]. Disponível em: http://sisaps.saude.gov.br/sisvan/relatoriopublico/index

12. Rosaneli CF, Baena CP, Auler F, Nakashima ATA, Netto-Oliveira ER, Oliveira AB, et al. Aumento da pressão arterial e obesidade na infância: uma avaliação transversal de 4.609 escolares. Arq Bras Cardiol 2014;103(3):238-44. https://doi. org/10.5935/abc.20140104

13. Kelly RK, Magnussen CG, Sabin MA, Cheung M, Juonala M. Development of hypertension in overweight adolescents: a review. Adolesc Health Med Ther 2015;6:171-87. https://doi.org/10.2147/AHMT.S55837

14. Brasil. Ministério da Saúde. Secretaria de Atenção à Saúde. Departamento de Atenção Básica. Norma Técnica do Sistema de Vigilância Alimentar e Nutricional. Orientações para a coleta e análise de dados antropométricos em serviços de saúde. Brasília: Ministério da Saúde; 2011. [acessado em 16 nov. 2021]. Disponível em: https://bvsms.saude.gov.br/bvs/publicacoes/ orientacoes_coleta_analise_dados_antropometricos.pdf.

15. Sardinha LB, Santos DA, Silva AM, Grøntved A, Andersen LB, Ekelund U. A comparison between BMI, waist circumference, and waist-to-height ratio for identifying cardio-metabolic risk in children and adolescents. PLoS One 2016;11(2):e0149351. https://doi.org/10.1371/journal.pone.0149351

16. Wang Y, Howard AG, Adair LS, Wang H, Avery CL, Gordon-Larsen P. Waist circumference change is associated with blood pressure change independent of BMI change. Obesity (Silver Spring) 2020;28(1):146-53. https://doi.org/10.1002/oby.22638

17. Cruz NRC, Cardoso PC, Frossard TNSV, Ferreira FO, Brener S, Gomides AFF, et al. Waist circumference as high blood pressure predictor in school age children. Cien Saude Colet 2019;24(5):1885-93. https://doi.org/10.1590/141381232018245.18012017

18. Zhang YX, Zhao JS, Chu ZH. Children and adolescents with low body mass index but large waist circumference remain high risk of elevated blood pressure. Int J Cardiol 2016;215:23-5. https://doi.org/10.1016/j.ijcard.2016.04.040

19. Parker ED, Sinaiko AR, Kharbanda EO, Margolis KL, Daley MF, Trower NK, et al. Change in weight status and development of Hypertension. Pediatrics 2016;137(3):e20151662. https://doi.org/10.1542/peds.2015-1662

20. Freedman DS, Serdula MK, Srinivasan SR, Berenson GS. Relation of circumferences and skinfold thicknesses to lipid and insulin concentrations in children and adolescents: the Bogalusa Heart Study. Am J Clin Nutr 1999;69(2):308-17. https://doi. org/10.1093/ajcn/69.2.308

21. National High Blood Pressure Education Program Working Group on High Blood Pressure in Children and Adolescents. The fourth report on the diagnosis, evaluation, and treatment of high blood pressure in children and adolescents. Pediatrics 2004;114(2 Suppl 4th Report):555-76. PMID: 15286277

22. Kuciene R, Dulskiene V. Associations between body mass index, waist circumference, waist-to-height ratio, and high blood pressure among adolescents: a cross-sectional study. Sci Rep. 2019;9(1):9493. https://doi.org/10.1038/s41598-019-45956-9

23. Choy CS, Chan WY, Chen TL, Shih CC, Wu LC, Liao CC. Waist circumference and risk of elevated blood pressure in children: a cross-sectional study. BMC Public Health 2011;11:613. https://doi.org/10.1186/1471-2458-11-613

24. Pazin DC, Rosaneli CF, Olandoski M, Oliveira ERN, Baena CP, Figueredo AS, et al. Waist circumference is associated with blood pressure in children with normal body mass index: a cross-sectional analysis of 3,417 school children. Arq Bras Cardiol 2017;109(6):509-15. https://doi.org/10.5935/abc.20170162 
25. Kuwahara E, Asakura K, Nishiwaki Y, Komatsu H, Nakazawa A, Ushiku H, et al. Steeper increases in body mass index during childhood correlate with blood pressure elevation in adolescence: a long-term follow-up study in a Japanese community. Hypertens Res 2014;37(2):179-84. https://doi.org/10.1038/hr.2013.109

26. Sabo RT, Lu Z, Daniels S, Sun SS. Serial childhood BMI and associations with adult hypertension and obesity: the Fels Longitudinal Study. Obesity (Silver Spring) 2012;20(8):1741-3. https://doi.org/10.1038/oby.2012.58

27. Fonseca MJ, Oliveira A, Azevedo I, Nunes J, Santos AC. Association of pubertal development with adiposity and cardiometabolic health in girls and boys-findings from the generation XXI birth cohort. J Adolesc Health 2019;65(4):558-63. https://doi.org/10.1016/j.jadohealth.2019.05.014

28. Chan NPT, Choi KC, Nelson EAS, Chan JC, KongAPS. Associations of pubertal stage and body mass index with cardiometabolic risk in Hong Kong Chinese children: a cross-sectional study. BMC Pediatr 2015;15:136. https://doi.org/10.1186/s12887-0150446-0

29. Kelishadi R, Mirmoghtadaee P, Najafi H, Keikha M. Systematic review on the association of abdominal obesity in children and adolescents with cardio-metabolic risk factors. J Res Med Sci 2015;20(3):294-307. PMID: 26109978

30. Kelly AS, Steinberger J, Jacobs DR, Hong CP, Moran A, Sinaiko AR. Predicting cardiovascular risk in young adulthood from the metabolic syndrome, its component risk factors, and a cluster score in childhood. Int J Pediatr Obes 2011;6(2-2):e283-9. https://doi.org/10.3109/17477166.2010.528765

31. Freedman DS, Goodman A, Contreras OA, DasMahapatra P, Srinivasan SR, Berenson GS. Secular trends in BMI and blood pressure among children and adolescents: the Bogalusa Heart Study. Pediatrics 2012;130(1):159-66. https://doi.org/10.1542/ peds.2011-3302

32. Flynn JT, Kaelber DC, Baker-Smith CM, Blowey D, Carroll AE, Daniels SR, et al. Clinical practice guideline for screening and management of high blood pressure in children and adolescents. Pediatrics 2017;140(3):e20171904. https://doi.org/10.1542/ peds.2017-1904

33. Malachias MVB, Souza WKSB, Plavnik FL, Rodrigues CIS, Brandão AA, Neves MFT, et al. Capítulo 10 - Hipertensão na Criança e no Adolescente. In: Malachias MVB, Souza WKSB, Plavnik FL, Rodrigues CIS, Brandão AA, Neves MFT, et al. $7^{a}$ Diretriz Brasileira de Hipertensão Arterial. Arq Bras Cardiol. 2016;107(Suppl.3):53-63. Disponível em: https://www.scielo.br/j/ abc/a/9fCp7wjFQhRYmqDbSTRvn3L/?format=pdf\&lang=pt

34. Sociedade Brasileira de Pediatria. Manual de Orientação. Hipertensão arterial na infância e adolescência. Rio de Janeiro: Departamento Científico de Nefrologia; 2019. Disponível em: https://www.sbp.com.br/fileadmin/user_upload/21635c-MO_-_ Hipertensao_Arterial_Infanc_e_Adolesc.pdf 